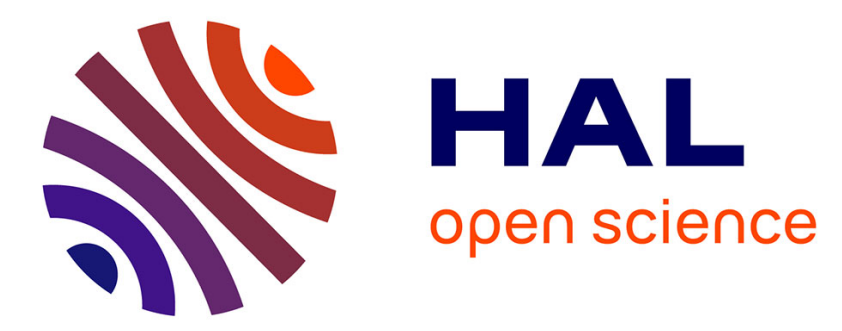

\title{
Impact of the primary particle polydispersity on the radiative properties of soot aggregates
}

Jerôme Yon, Franklin Liu, José Carlos Moran Cofré, A. Fuentes

\section{To cite this version:}

Jerôme Yon, Franklin Liu, José Carlos Moran Cofré, A. Fuentes. Impact of the primary particle polydispersity on the radiative properties of soot aggregates. Proceedings of the Combustion Institute, 2019, 37 (1), pp.1151-1159. 10.1016/j.proci.2018.07.065 . hal-01854803

\section{HAL Id: hal-01854803 \\ https://hal.science/hal-01854803}

Submitted on 21 Oct 2021

HAL is a multi-disciplinary open access archive for the deposit and dissemination of scientific research documents, whether they are published or not. The documents may come from teaching and research institutions in France or abroad, or from public or private research centers.
L'archive ouverte pluridisciplinaire HAL, est destinée au dépôt et à la diffusion de documents scientifiques de niveau recherche, publiés ou non, émanant des établissements d'enseignement et de recherche français ou étrangers, des laboratoires publics ou privés.

\section{(ㄷ)(1) $\$$}

Distributed under a Creative Commons Attribution - NonCommerciall 4.0 International 


\title{
Impact of the Primary Particle Polydisersity on the Radiative Properties of Soot Aggregates
}

\author{
J. Yon ${ }^{\mathrm{a}}$, F. Liu ${ }^{\mathrm{b}}$, J. Morán ${ }^{\mathrm{c}}$, A. Fuentes ${ }^{\mathrm{c}}$ \\ ${ }^{a}$ Normandie Univ, INSA Rouen, UNIROUEN, CNRS, CORIA, 76000 Rouen, France \\ ${ }^{b}$ Measurement Science and Standards, National Research Council of Canada, Ottawa, Ontario, Canada. \\ ${ }^{c}$ Departamento de Industrias, Universidad Técnica Federico Santa María, Av. España 1680, Casilla 110-V, Valparaíso, Chile.
}

\begin{abstract}
Combustion generated soot appears as fractal aggregates formed by polydisperse nearly spherical primary particles. Knowledge of their radiative properties is a prerequisite for laser based diagnostics of soot. In this parametric study, the effect of primary particle polydispersity on soot aggregate absorption and scattering properties is investigated numerically. Two series of fractal aggregates formed by normal and lognormal distributed primary particles of different levels of standard deviation were numerically generated for typical flame soot with a fractal dimension and prefactor fixed to $D_{f}=1.73$ and $k_{f} \approx 1.5$, respectively. Three aggregate sizes consisting of $N_{p}=15,50$ and 150 monomers per aggregate were investigated. Due to the uncertainty in soot refractive index, radiative properties were calculated by considering two different refractive indices at $\lambda \approx 532 \mathrm{~nm}$ recommended in the literature using the Discrete Dipoles Approximation and the Generalized multi-sphere Mie method. The results are interpreted in terms of correction factors to the Rayleigh-Debye-Gans theory for fractal aggregates (RDG-FA) for the forward scattering cross section $A$ and for the absorption cross section $h$. It is shown that differential cross section for vertically polarized incident light, total scattering and absorption cross sections are well predicted by the RDG-FA theory for all considered aggregates formed by normally $\left(\sigma / \bar{d}_{p} \leq 30 \%\right)$ and lognormally $\left(\sigma_{g e o} \leq 1.6\right)$ distributed primary particles. The refractive index is found to be of greater impact than primary particle polydispersity on the importance of multiple scattering. The radiative force per unit laser power experienced by the soot aggregates was found primarily determined by the aggregate volume, regardless of the level of primary particle polydispersity.
\end{abstract}

Keywords: Radiative properties of soot aggregates, Polydisperse primary particles, Discrete dipole approximation, Generalized multi-sphere Mie, laser light scattering by soot

Email address: yon@coria.fr (J. Yon) 


\section{Introduction}

Accurate knowledge of the optical properties of soot aerosol is essential to interpret the detected signals in terms of soot properties, such as concentration, primary particle (PP) and aggregate sizes, in laser based measurements, to assess the role of soot aerosol in atmospheric chemistry and combustion processes. In this context it is highly desirable to develop relatively simple yet sufficiently accurate models for the radiative properties of fractal-like soot aggregates. The RayleighDebye-Gans theory for fractal aggregates (RDG-FA) is very simple to use but suffers some drawbacks and poor accuracies under certain conditions, mainly due to the neglect of internal coupling or multiple scattering within an aggregate.

Many studies have been performed to evaluate the accuracy of RDG-FA theory $[1,2]$. In addition, a few studies have been carried out to introduce correction factors or to propose some alternatives to the RDG-FA theory to extend its applicability and/or improve its accuracy. In almost all light scattering experiments, the results were interpreted using the RDG-FA theory assuming that soot aggregates are formed by monodisperse PP in point-contact, though in reality these particles are formed by polydisperse PP with a certain degree of overlapping. In consequence, some efforts have been attempted to investigate the effects of different parameters on soot radiative properties, such as PP polydispersity [3-6], number of PP per aggregate and wavelengths [7], necking and overlapping [8]. Although it has been demonstrated that the PP polydispersity has a significant impact on soot radiative properties [9-11], there is currently a lack of adequate quantitative understanding of the consequence of applying the RDG-FA theory to soot aggregates formed by polydisperse PP and only a few relevant studies have been conducted.

Farias et al. [3] used the Iskander-Chen-Penner (ICP) method to compare numerical results of RDG-FA for normal primary particle size distribution (NPPSD). The authors observed that the forward and total scattering cross sections are increased by an amount of 14 and $41 \%$, respectively, for $\sigma / \overline{d_{p}}=0.15$ and 0.25 . They also demonstrated that this effect can be reasonably predicted by RDG-FA if the volume equivalent PP size is considered. However, the assumptions on ICP method limit the validity of their results. Liu et al. [4] used the Multi Sphere T-matrix method to study the radiative properties of aggregates consisting of lognormal primary particle size distribution (LNPPSD) with $\sigma_{\text {geo }} \leq 1.5$. They found that RDG-FA is significantly challenged for approximating the scattering and absorp- tion cross sections of the aggregates with polydisperse PP at $\lambda=650 \mathrm{~nm}$ and a complex refractive index $m=1.75+i 0.435$. Finally, Wu et al. [5] used the Superposition T-matrix method to calculate the radiative properties of aggregates for LNPPSD $\left(\sigma_{g e o} \leq 4.0\right)$, $\lambda=550 \mathrm{~nm}$, and $m=1.95+i 0.79$ and found only a slight effect $(<10 \%)$ on the extinction and absorption cross sections regarding PP polydispersity. Nevertheless, no attempt was made to extend RDG-FA to fractal aggregates formed by polydisperse PP.

Regarding primary particle size distribution (PPSD) of soot, experimental studies have also found that it can be well described by a LNPPSD or a NPPSD [12, 13]. Available experimental studies have reported a maximum polydispersity of $\sigma / \overline{d_{p}}=30 \%$ (the standard deviation) and $\sigma_{\text {geo }}=1.6$ (the geometric standard deviation) for NPPSD [14] and LNPPSD [15], respectively. Additionally, the effect of PPSD has been the subject of studies for fractal aggregate physical properties other than radiative, for instance structure and mobility $[13,16]$.

In this parametric study, we investigated the effects of PP polydispersity on the radiative properties of soot aggregates at 550 and $532 \mathrm{~nm}$, which are typical wavelengths used in light scattering experiments, following the recent studies of Liu et al. [4] and Wu et al. [5]. This research and previous numerical studies form the indispensable prerequisite to take into account PP polydispersity in the retrieval of soot aggregate properties based on light scattering experiments. The objectives of this study are threefold: (1) to investigate the effect of PP polydispersity on fractal aggregate radiative properties under conditions relevant to flame soot, (2) to propose and evaluate corrections to the RDG-FA theory for calculations of radiative properties of soot aggregates formed by polydisperse PPs, and (3) to assess the effect of PP polydispersity on the radiative force experienced by soot aggregates due to laser irradiance.

\section{Numerical Methods}

\subsection{Generation of fractal-like aggregates}

Fractal aggregates were generated numerically by using the Flage particle-cluster aggregation algorithm described by Skorupski et al. [17]. The morphology of these aggregates is considered to be fractal-like and described as $N_{p}=k_{f}\left(R_{g} / R_{p}\right)^{D_{f}}$, where $N_{p}$ and $R_{p}$ correspond to the number and average (arithmetic or geometric for NPPSD and LNPPSD, respectively) radius of primary particles, respectively, and $R_{g}$ is the radius of gyration calculated as proposed by Dastanpour et al. [13]. 
$D_{f}$ and $k_{f}$ stand for the fractal dimension and prefactor, respectively. Because of its simplicity, capability of generating aggregates of prescribed fractal parameters, and efficiency, the tunable algorithms have been widely used to generate fractal aggregates for study of their radiative properties $[4,18,19]$. In the present study, concerning the specific role played by PP polydispersity, the fractal dimension is retained approximately constant $\left(D_{f} \approx 1.73\right)$ for all levels of PP polydispersity. The fractal dimension is selected to be representative of soot produced from miniCAST [20]. However, the corresponding prefactor, observed to be $\approx 1.9$ from TEM image analysis, is slightly reduced because overlapping is not taken into account in the present study [8]. This reduction is based on the extrapolation of the relationship found by Brasil et al. [21] between $k_{f}$ and overlapping, who proposed $k_{f}=1.5$ without overlapping. It was also found that for $\sigma_{g e o}>1.6$ the prefactor experiences a small decrease (from $k_{f}=1.50$ ) with increasing level of PP polydispersity. As found by Eggersdorfer and Pratsinis [16], for $\sigma_{\text {geo }}>1.6$ the structure of fractal aggregates (regardless of the aggregation mechanism) is considerably affected by PP polydispersity. It is recognized that the tunable method used to generate fractal aggregates in this study does not correspond to particle aggregation via any physical or chemical aggregation mechanisms (including for example surface growth) that could link the PP polydispersity to the fractal-like morphology [16]. On the other hand, tunable algorithms offer the advantages to investigate the effect of a single parameter, such as PP polydispersity, while keeping all other parameters constant. The performance of such modeling in general involves tuning of model parameters given the current lack of detailed understanding of the soot surface growth process. Moreover, the studies of Bushell and Amal [9] and Eggersdorfer and Pratsinis [16] showed that the fractal dimension remains largely unchanged, though the prefactor decreases, with increasing the level of PP polydispersity (up to $\sigma_{\text {geo }} \approx 1.5$ ). For these reasons, we chose to adopt the tunable method that permits to isolate the effect of PP polydispersity and we considered a realistic maximum $\sigma_{\text {geo }}$ of 1.6 for the evaluation of the radiative properties. In addition, the strategy chosen in the present study consists in increasing the PP polydispersity while keeping the mean radius (arithmetic or geometric for NPPSD and LNPPSD, respectively) constant. Consequently, the increase of PP polydispersity impacts the aggregate volume. This effect is illustrated in Fig. 1, where $a_{e f f}$ is the radius of the equivalent sphere having the same volume as the aggregate $\left(V_{a g g}=4 \pi a_{a e f f}^{3} / 3\right)$. Figure 1 shows that the aggregates formed by LNPPSD with $\sigma_{g e o}>2$ may have larger levels of PP polydispersity than those formed by NPPSD. In consequence, the volume is more affected for aggregates in the LNPPSD series than those in the NPPSD series. Nevertheless, it is noticed that the fairly high levels of PP polydispersity $\left(S T D\left(R_{p}\right) / R_{p}>0.4\right)$ are typically not encountered in flame-generated soot aggregates. Aggregates formed by highly polydisperse PPs, with $\sigma_{\text {geo }}>1.6$ are only considered for the calculation of the radiative force in the present study. For lower levels of PP polydispersity, relatively small increase of $a_{e f f}$ is observed in comparison to the change in the number of primary particles.

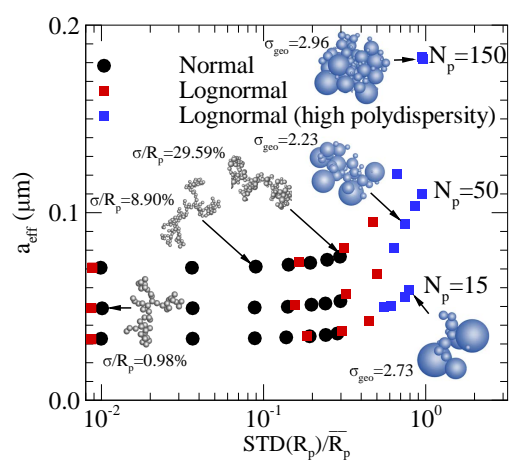

Figure 1: Impact of the PP polydispersity on the aggregate volume equivalent radius. The standard deviation is indicated in the horizontal axis, $\sigma_{\text {geo }}$ is shown as legend.

\subsection{The discrete dipole approximation}

The radiative properties of soot aggregates were calculated by using the Discrete Dipole Approximation (DDA) through the DDSCAT code (version 7.3.2) [22]. This method offers the advantage for dealing with particles of complex shapes or morphologies. The principle of DDA is to discretize the particle into a finite array of polarizable points (dipoles) and to consider their interactions with the local electric field. It is equivalent to the volume integral equation for the electric field. In this study, the dipole density was fixed at 15 (corresponding to 15 dipoles per mass equivalent diameter). The present study concerns the visible domain of the spectrum. Since numerous light scattering experiments have been done with green lasers, $\lambda=532 \mathrm{~nm}$ is selected in the present study.

The considered optical index $m_{1}=1.5+i 0.62$ was reported by Bescond et al. [20] for miniCAST soot characterized by a low amount of organic compounds $(\mathrm{OC} / \mathrm{TC}=4.1 \%)$ and high degree of graphitization. It 
was determined by interpreting extinction spectra measurements based on a modified RDG-FA theory by applying some correction factors regarding the multiple scattering effects, using the Drude-Lorentz dispersion law, by considering the size distribution, bulk density and specific morphological properties. It is noticed that the optical index of soot generated under the same conditions was previously reported to be $m=1.66+i 0.76$. However, it was obtained with a simpler size distribution, neglect of multiple scattering effect, and without measurement of the bulk density. Nevertheless, the optical index of soot is subject to uncertainties, depending on the flame conditions and the experimental and analysis methods. To evaluate the influence of refractive index, calculations are also conducted with another refractive index $m_{2}=1.95+i 0.79$ determined by Smith et al. [23] for annealed amorphous carbon films and recommended to be used at similar wavelengths (550 $\mathrm{nm}$ ) by Bond and Bergstrom [24]. Finally, to determine the aggregate structure factors without multiple scattering additional calculations were performed using $m=1.01+i 0.01$. The criterion $|m| k d<0.5$ for accurate calculations of the radiative properties using DDA [22] is ensured in this study $(|m| k d<0.12)$. The radiative properties of aggregates calculated by DDA were averaged over 1000 orientations.

\subsection{Validation of the DDA results}

To validate the results of DDA, calculations were also carried out using the numerically exact Generalized Multi-sphere Mie Solution method (GMM). GMM is an extension of Mie theory for solving exactly the Maxwell's equations for a particle system containing non-overlapping spheres. GMM results were obtained with averaging over more than 15,000 orientations. Ta-

Table 1: Comparison between DDA and GMM results. Average relative errors calculated as: $\epsilon_{X}=100 \cdot\left(X_{D D A}-X_{G M M}\right) / X_{G M M}$.

\begin{tabular}{cccccc}
\hline \multirow{2}{*}{$N_{p}$} & \multirow{2}{*}{$X$} & \multicolumn{2}{c}{ LNPPSD } & \multicolumn{2}{c}{ NPPSD } \\
& & $\lambda_{532}$ & $\lambda_{550}$ & $\lambda_{532}$ & $\lambda_{550}$ \\
\hline \multirow{3}{*}{15} & $C_{h h} / C_{v v}$ & $-0,039$ & $-0,015$ & 0,007 & 0,157 \\
& $C_{v h} / C_{v v}$ & $-3,645$ & $-0,874$ & 0,415 & 3,758 \\
& $C_{a b s}$ & 1,111 & 4,678 & 0,547 & 4,411 \\
\hline \multirow{3}{*}{50} & $C_{h h} / C_{v v}$ & $-1,261$ & $-2,048$ & $-0,144$ & $-0,145$ \\
& $C_{v h} / C_{v v}$ & $-5,480$ & $-4,684$ & 4,294 & 6,877 \\
& $C_{a b s}$ & 0,947 & 4,338 & 1,232 & 5,100 \\
\hline \multirow{3}{*}{150} & $C_{h h} / C_{v v}$ & 1,193 & 2,631 & $-0,234$ & $-0,298$ \\
& $C_{v h} / C_{v v}$ & 5,156 & 7,821 & 2,584 & 4,264 \\
& $C_{a b s}$ & 0,945 & 4,406 & 1,111 & 4,872 \\
\hline \multirow{4}{*}{} & & & & &
\end{tabular}

ble 1 shows the relative error $\epsilon_{X}=100 \cdot\left(X_{D D A}-\right.$
$\left.X_{G M M}\right) / X_{G M M}$ of the DDA results benchmarked by those of GMM. The error was calculated for three variables $(X)$ : (1) the ratio of polarized angular scattering cross sections $C_{h h} / C_{v v}$ (here $C_{h h}$ denotes the differential scattering cross section for horizontally incident and horizontally scattering polarized light, other quantities are similarly defined), (2) $C_{v h} / C_{v v}$, and (3) the absorption cross section $C_{a b s}$ for the three aggregate sizes with different levels of PP polydispersity. The agreement between DDA and GMM is considered very good with only small deviations $\left(\epsilon_{X}<8 \%\right.$ for the two ratios and $\epsilon_{C_{a b s}}<6 \%$ for the absorption cross section). It is noticed that when comparing the $C_{h h} / C_{v v}$ and $C_{v h} / C_{v v}$ ratios, the relatively large relative errors only occur for $C_{v h} / C_{v v}$, but not for $C_{h h} / C_{v v}$. This is because the former is generally very small. Nevertheless, the DDA results are considered sufficiently accurate for the purposes of this study.

\section{Results and discussion}

The structure factor without multiple scattering is presented in Fig. 2(a) and (b). It represents the angular dependency of the vertically polarized scattered light by the aggregates relative to the vertically polarized incident light. It corresponds to the Fourier transform of the pair-correlation function and bears the morphological information of the particle. In the present case, it is obtained by dividing the $C_{v v}^{a g g}$ calculated by DDA with $m=1.01+i 0.01$ by the product of the primary particle $C_{v v}^{p}$ based on Rayleigh theory and $N_{p}^{2}$. For each aggregate, the PP differential scattering cross section $C_{v v}^{p}$ is based on the Rayleigh approximation of a sphere having a volumetric mean radius $\left(a_{p 3}=a_{e f f} \cdot N_{p}^{-1 / 3}\right)$. Indeed, in the forward scattering the RDG-theory stipulates that the scattered intensity is proportional to $V_{a g g}^{2}$. This methodology enables a good determination of the theoretical value of unity in forward scattering at $q R_{g}=0$. We can distinguish the Guinier regime of small values of $q R_{g}$ less than unity (exponential decrease, with $q=4 \pi \sin (\theta / 2) / \lambda)$ from the power-law regime of large values of $q R_{g}$ (larger than unity). The structure factor of Dobbins and Megaridis for fractal aggregates [25] is reported as black dashed lines with $D_{f}=1.73$ initially imposed during the aggregate generation. For both considered series of aggregates, corresponding to primary particles of NPPSD and LNPPSD, the Guinier regime is well observed, though the decrease is enhanced for large $\sigma_{g e o}$ of the LNPPSD series. For $q R_{g}>1.62$, based on statistics of a large ensemble of aggregates, a linear behavior is expected with a slope supposed to be $-D_{f}$. For individual aggregates formed by NPPSD and those 

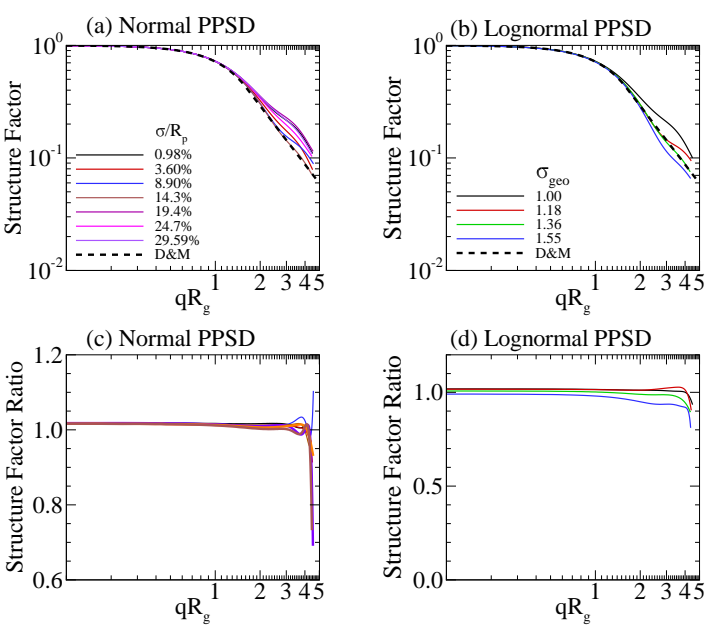

Figure 2: Figs. (a) and (b) present the aggregate structure factors (without internal coupling) obtained at $\lambda=532 \mathrm{~nm}$ with $m=1.01+$ $i 0.01$ for NPPSD and LNPPSD. The dashed lines are the structure factor of Dobbins and Megaridis. Figs. (c) and (d) present the ratio between angular light scattering patterns computed with (using $m=$ $1.5+i 0.62$ ) and without multiple scattering for aggregates of NPPSD and LNPPSD series at $\lambda=532 \mathrm{~nm}$ and $N_{p}=150$.

formed by LNPPSD of low levels of polydispersity, this behavior is essentially preserved with an apparent individual fractal dimension slightly different from that initially imposed $\left(D_{f}\right)$.

Nevertheless, for the range of PP polydispersity relevant to flame soot $\left(\sigma_{g e o}<1.6\right)$ the fractal behavior can largely be ensured.

Let us consider now the structure factors calculated using the assumed refractive indexes of soot at $532 \mathrm{~nm}$ for aggregates of both series. By using $m=1.5+i 0.62$, the internal coupling or multiple scattering within aggregates is automatically considered in DDA calculations. To highlight the effect of internal coupling, Figs. 2(c) and 2(d) present the ratio between both the structure factors with and without multiple scattering. This ratio is useful to develop corrections to the RDGFA theory to improve its accuracy for the calculation of vertical-vertical differential scattering cross section $\left(C_{v v}\right)$ of soot aggregates formed by polydisperse PP. In Figs. 2(c) and 2(d) we can observe that, for the considered wavelength and refractive index, the ratios remain at almost unity for aggregates formed by NPPSD and for those formed by LNPPSD except for backscattering at large $q R_{g}$, indicating a good prediction by the RDGFA theory.

The correction factor required to bring the RDG-FA results to those of DDA for the prediction of the forward scattering $A$ corresponds to the ratios of forward scattering shown in Figs. 2(c) and 2(d) at $q R_{g}=0$. Its repre- sentation as a function of the standard deviation does not display a clear trend (see supplementary material that also contains results for larger geometric standard deviations). Contrarily, its representation as a function of the equivalent radius $a_{e f f}$ shows a more clear trend, regardless of the aggregate size or the level of PP polydispersity (Fig. 3(a)). This leads to a conclusion that, for a given optical index, the aggregate size or volume has a stronger impact on $A$ than PP polydispersity itself. To identify the sensitivity of A to PP polydispersity, we also investigated the corresponding ratios for aggregates formed by monodisperse PP for $N_{p}=50$ by varying the primary particle size (dashed lines in Fig. 3) and by increasing $N_{p}$ at fixed $D_{p}$ (black dotted lines) based on previously published results [7] (obtained with $\left.m_{3}=1.61+i 0.74\right)$. The comparison of the tree curves indicates that, for a given aggregate volume, the deviation of $A$ from unity is enhanced by the increase of the PP diameter and even more when the polydispersity increases (presence of very large primary spheres in the aggregate). Nevertheless, the optical index seems to play a predominant role (the small difference in wavelength between 532 and $550 \mathrm{~nm}$ is not expected to have a large impact on the ratio). The refractive index proposed by Smith et al. [23] induces a stronger multiple scattering effect, due to the larger deviations from unity, even without PP polydispersity (small $a_{\text {eff }}$ ). This effect is mainly due to the larger real part of the optical index as recently shown in [26]. With regard to the scattering function $F(m)$, the value of Smith et al. $\left(F\left(m_{2}\right)=0.39\right)$ is 1.7 times that of the refractive index of miniCAST $\operatorname{soot}\left(F\left(m_{1}\right)=0.23\right)$. Thus, stronger scattering and multiple scattering effects are expected when using the refractive index of Smith et al.

Figure 3(b) presents the corrections to bring the RDG-FA theory to the DDA results for aggregate absorption cross section. Similar trends between corrections to the absorption cross section and the forward scattering cross section can be observed and this point has already been found in previous studies [6-8, 26, 27].

Figures 4(a) and 4(b) report the ratio $C_{h h} / C_{v v}$, which is theoretically equal to $\cos ^{2}(\theta)$ in the RDG-FA theory. A good agreement between the results of DDA and RDG-FA is observed. However, such agreement deteriorates slightly with increasing level of PP polydispersity, especially for LNPPSD around $90^{\circ}$. Once again, the multiple scattering effect (internal coupling among primary particles) is suspected to cause the deviations. This conjecture is supported by Figs. 4(c) and 4(d) representing the depolarization ratio, i.e., $C_{v h} / C_{v v}$. This ratio is supposed to be null in the RDG-FA theory 

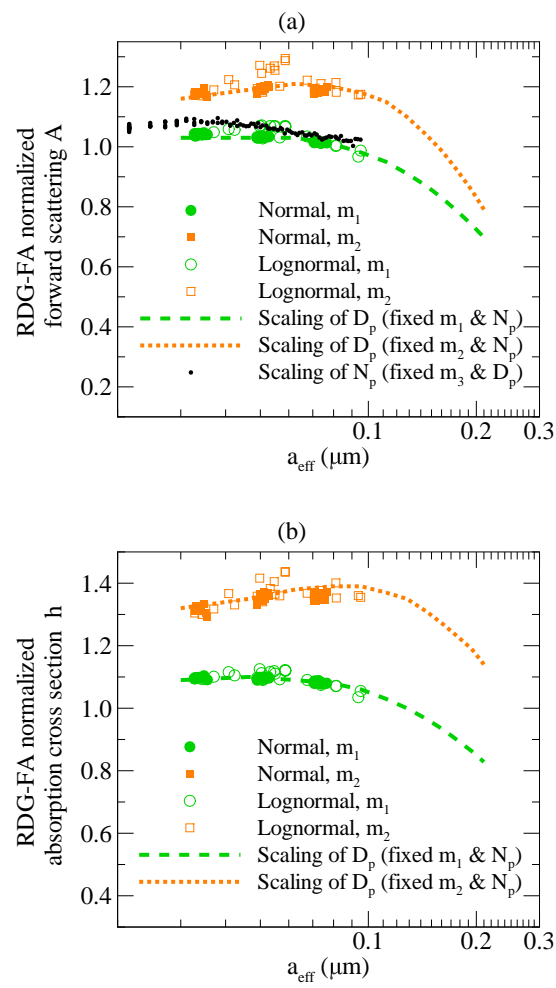

Figure 3: Corrections to bring the RDG-FA theory for the forward scattering and absorption cross sections for all the aggregates considered at $m_{1}=1.5+i 0.62\left(F\left(m_{1}\right)=0.23\right), m_{2}=1.95+i 0.79$ $\left(F\left(m_{2}\right)=0.39\right)$ and $m_{3}=1.61+i 0.74$ : (a) the correction A for forward scattering cross section and (b) the correction $\mathrm{h}$ for absorption cross section. The dashed lines are for results obtained for scaled aggregates formed by monodisperse PP and the black dotted line corresponds to the variation of $N_{p}$ based on the results by [7]

and is shown to generally increase with scattering angle [28]. The present study reveals that the depolarization is not strongly affected by the degree of PP polydispersity. The anisotropy coefficient $A_{13}$ of each aggregate was calculated based on the eigenvalues of the inertia tensor as proposed by Heison et al. [29] and adapted to polydisperse monomers [30]. The values are reported in the legend of Figure 4(a) and (b). It is noticed that the increase in depolarization in backward scattering directions (scattering angles close to $180^{\circ}$ ) has often been attributed to an increase of the particle anisotropy. In the present case, even at very small levels of polydispersity, a slight increase of the depolarization ratio is observed without increasing the particle anisotropy.

Integration of the differential scattering cross section over $4 \pi s r$ permits to evaluate the effects of PP polydispersity on the total scattering cross section $\sigma_{s c a}^{a g g}$. According to the RDG-FA theory, $\sigma_{\text {sca }}^{a g g}=N_{p}^{2} \sigma_{\text {sca }}^{p p} g$, here $g$ is the aggregate total scattering factor and can be determined from the DDA results. It is interesting to compare such determined $g_{D D A}$ to the widely used factor in the RDG-FA theory $g_{\text {Dobbins }}$ [25]. Previous similar investigations concluded that the accordance between the two factors is good if $g_{\text {Dobbins }}$ is multiplied by the correction for the forward scattering (cf. Fig. 3(a)). Because $g_{\text {Dobbins }}$ has been obtained by integrating the Dobbins and Megaridis expression of the structure factor (plain curves in Fig. 2). This assumption holds as long as structure factor remains essentially the same. In order to see if this finding holds for aggregates formed by polydisperse PP, we report in Fig. 5 the ratio between $g_{D D A}$ (numerically accurate evaluation) and $A g_{\text {Dobbins }}$, i.e., the corrected RDG-FA expression. The deviations of this ratio from unity indicate the error caused by RDG-FA theory even though part of the multiple scattering effects is taken into account through the correction factor $A$. The obtained ratios are scattered but remain around unity for the considered range of PP polydispersity and decrease slightly with increasing the PP polydispersity. Nevertheless, it is important to notice that for the range of PP polydispersity typically encountered in flame-generated soot $\left(S T D\left(R_{p}\right) / R_{p}<0.4\right)$, it appears that the RDG-FA theory, once corrected by the $A$ factor, is able to predict fairly accurate aggregate total scattering cross sections. This is shown to be true for both optical indices considered.

Finally, we report in Fig. 6 the radiative force experienced by a soot aggregate due to laser irradiance per unit energy density (radiative pressure cross section $C_{p r}=C_{e x t}-\tilde{g} C_{s c a}$ where $\tilde{g}$ represents the asymmetry parameter [22, 31]). This quantity can be of interest not only for optical manipulation of soot material [32], but also for other fields of science, such as astrophysics concerns for interstellar grains [31]. It can be used for the evaluation of the photopheretic force caused by a Planck radiation source [33].

It is interesting to observe a nearly power law relationship between that radiative force and the volume equivalent radius. This is also true for larger LN polydisersities (up to $\sigma_{g e o}=3.0$ ), reported in blue boxes in Fig. 6. The exponent of that power-law is about 2.97 , suggesting that radiative force is essentially governed by the absorption cross section (proportional to the aggregate volume) and thus weakly affected by the PP polydispersity (except for very large geometric standard deviations). This was confirmed by comparing the radiative cross sections of the LNPPSD series for the largest aggregates $\left(N_{p}=150\right)$ by scaling the aggregates in order to achieve the same volume $\left(a_{\text {eff }}=0.1 \mu \mathrm{m}\right)$. It results in a relative standard deviation of $C_{p r}$ of $1 \%$ 

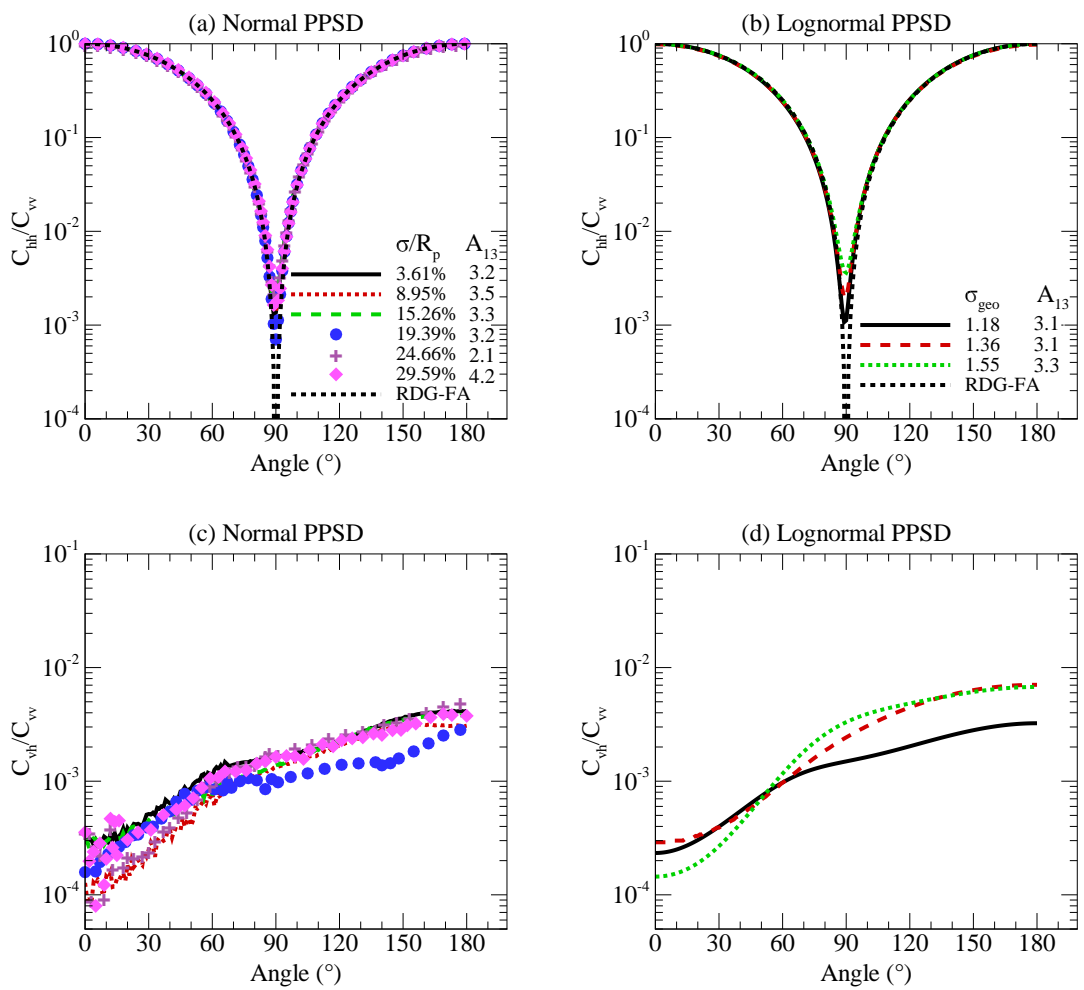

Figure 4: Impact of the PPSD on the $C_{h h} / C_{v v}$ and $C_{v h} / C_{v v}$ cross sections at $\lambda=532 \mathrm{~nm}$ and $m=1.5+i 0.62 . A_{13}$ stand for the anisotropy coefficient of each aggregate.

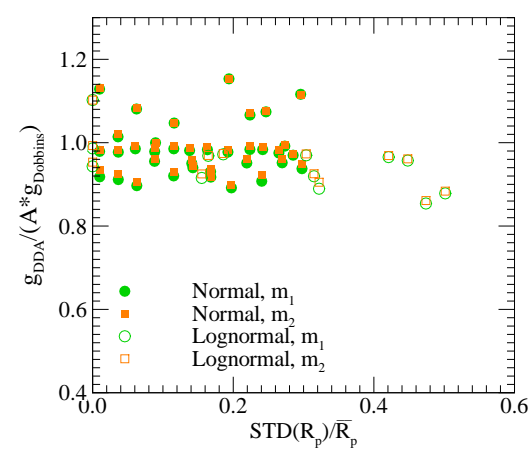

Figure 5: Impact of PPSD on the corrections applied in the RDGFA theory for the evaluation of the total scattering cross section $\sigma_{s c a}^{a g g}$, $m_{1}=1.5+i 0.62$ and $m_{2}=1.95+i 0.79$.

and $5 \%$ for $m_{1}=1.5+i 0.62$ and $m_{2}=1.95+i 0.79$, respectively.

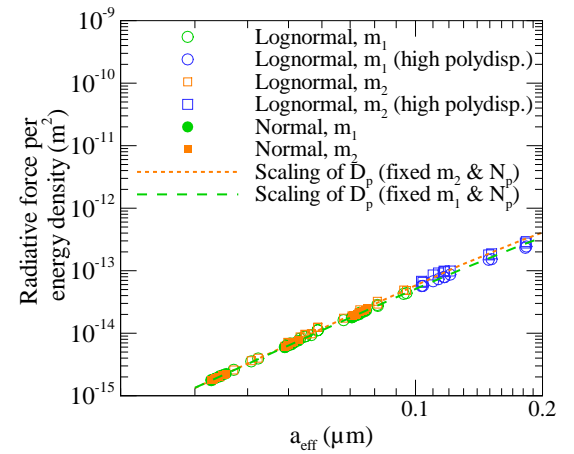

Figure 6: Radiative forcing per unit energy density. The dashed lines denote results obtained for aggregates formed by scaled monodisperse primary particles with $m_{1}=1.5+i 0.62$ and $m_{2}=1.95+i 0.79$.

\section{Conclusions}

The impact of primary particle polydispersity on the radiative properties of soot aggregates was numerically investigated using the discrete dipole approximation by considering two different refractive indices at $532 \mathrm{~nm}$ 
recommended in the literature to demonstrate the effect of uncertainty in soot refractive index on soot radiative properties. This study intents to gain better understanding of the importance of primary particle polydispersity. Such knowledge is essential to extend the current capabilities of light scattering experiments to retrieve more realistic soot aggregate morphological parameters by taking into account the polydispersity of primary particles. Calculations were conducted for both normal and lognormal primary particle size distributions. Fractal aggregates formed by polydisperse spherical primary particles in point-contact were generated by using the tunable Flage code with fractal parameters fixed to reported standard flame soot values. It has been shown that the fractal behavior is not significantly affected by PP polydispersities, for the range concerned in flame conditions.

The results are analyzed in terms of the correction factors required to bring the RDG-FA results to agree with those of DDA for forward scattering and absorption cross sections. The main findings of this study are that for primary particle polydispersity levels relevant to flame-generated soot, the RDG-FA theory is adequate when the volume mean primary particle diameter is used. The validity range of corrections proposed is dominated by internal multiple scattering and the magnitude of this effect is mainly influenced by the refractive index, both the aggregate size and primary particle polydispersity are of second order effect. This is true for angular light scattering $C_{v v}, C_{h h}$, absorption cross section $c_{a b s}$, and total light scattering cross section $c_{s c a}$. Only the depolarization ratio $C_{v h} / C_{v v}$ seems to be slightly increased in the case of lognormal distribution. Finally, the radiative force acting on soot particles is evaluated. For the considered two PPSDs, the radiative force is nearly proportional to the particle volume.

\section{Acknowledgments}

The authors thank the CRIANN for the computational resources provided by the Haute-Normandie region. A. Fuentes also acknowledges the Chilean CONICYT Research program under FONDECYT project 1161453.

\section{References}

[1] F. Liu, D. Snelling, in: 40th Thermophysics Conf., p. 4362.

[2] T. Farias, Ü. Köylü, M. Carvalho, Appl. Opt. 35 (1996) 65606567.

[3] T. L. Farias, Ü. Ö. Köylü, M. d. G. Carvalho, J. Quant. Spectrosc. Radiat. Transfer 55 (1996) 357-371.
[4] C. Liu, Y. Yin, F. Hu, H. Jin, C. M. Sorensen, Aerosol Sci. Tech. 49 (2015) 928-940

[5] Y. Wu, T. Cheng, L. Zheng, H. Chen, Aerosol Sci. Tech. 49 (2015) 941-949.

[6] J. Yon, C. Rozé, T. Girasole, A. Coppalle, L. Méès, Part. Part. Syst. Char. 25 (2008) 54-67.

[7] J. Yon, F. Liu, A. Bescond, C. Caumont-Prim, C. Rozé, F. X. Ouf, A. Coppalle, J. Quant. Spectrosc. Radiat. Transfer 133 (2014) 374-381.

[8] J. Yon, A. Bescond, F. Liu, J. Quant. Spectrosc. Radiat. Transfer 162 (2015) 197-206.

[9] G. Bushell, R. Amal, J. Raper, Part. Part. Syst. Char. 15 (1998) 3-8.

[10] J. Yin, L. Liu, J. Quant. Spectrosc. Radiat. Transfer 111 (2010) 2115-2126.

[11] N. Doner, F. Liu, J. Quant. Spectrosc. Radiat. Transfer 187 (2017) $10-19$.

[12] A. Bescond, J. Yon, F. Ouf, D. Ferry, D. Delhaye, D. Gaffié, A. Coppalle, C. Rozé, Aerosol Sci. Tech. 48 (2014) 831-841.

[13] R. Dastanpour, S. N. Rogak, J. Aerosol Sci. 94 (2016) 22-32.

[14] M. Wentzel, H. Gorzawski, K.-H. Naumann, H. Saathoff, S. Weinbruch, J. Aerosol Sci. 34 (2003) 1347 - 1370. Intercomparison of Soot Measurement Techniques.

[15] R. K. Chakrabarty, H. Moosmüller, M. A. Garro, W. P. Arnott, J. Walker, R. A. Susott, R. E. Babbitt, C. E. Wold, E. N. Lincoln, W. M. Hao, J. Geophys. Res. Atmos. 111 (2006).

[16] M. Eggersdorfer, S. Pratsinis, Aerosol Sci. Tech. 46 (2012) 347353.

[17] K. Skorupski, J. Mroczka, T. Wriedt, N. Riefler, Physica A 404 (2014) 106-117.

[18] A. Filippov, M. Zurita, D. Rosner, J. Colloid Interf. Sci. 229 (2000) 261-273.

[19] F. Liu, M. Yang, F. A. Hill, D. R. Snelling, G. J. Smallwood, Appl. Phys. B-Lasers O. 83 (2006) 383-395.

[20] A. Bescond, J. Yon, F.-X. Ouf, C. Rozé, A. Coppalle, P. Parent, D. Ferry, C. Laffon, J. Aerosol Sci. 101 (2016) 118-132.

[21] A. Brasil, T. Farias, M. Carvalho, J. Aerosol Sci. 30 (1999) 1379.

[22] B. T. Draine, P. J. Flatau, J. Opt. Soc. Am. A 11 (1994) 14911499.

[23] F. Smith, J. Appl. Phys. 55 (1984) 764-771.

[24] T. C. Bond, R. W. Bergstrom, Aerosol Sci. Tech. 40 (2006) 2767.

[25] R. A. Dobbins, C. M. Megaridis, Appl. Opt. 30 (1991) 47474754.

[26] C. M. Sorensen, J. Yon, F. Liu, J. Maughan, W. R. Heinson, M. J. Berg, J. Quant. Spectrosc. Radiat. Transfer 186 (2018) 89-101.

[27] F. Liu, J. Yon, A. Bescond, J. Quant. Spectrosc. Radiat. Transfer 172 (2016) 134-145.

[28] A. Bescond, J. Yon, T. Girasole, C. Jouen, C. Rozé, A. Coppalle, J. Quant. Spectrosc. Radiat. Transfer 126 (2013) 130-139.

[29] W. Heinson, C. Sorensen, A. Chakrabarti, J. Colloid Interf. Sci. 375 (2012) 65-69.

[30] M. L. Eggersdorfer, S. E. Pratsinis, Adv. Powder Technol. 25 (2014) 71-90.

[31] A. G. Hoekstra, M. Frijlink, L. Waters, P. Sloot, J. Opt. Soc. Am. A 18 (2001) 1944-1953.

[32] V. Karasev, N. Ivanova, A. Sadykova, N. Kukhareva, A. Baklanov, A. Onischuk, F. Kovalev, S. Beresnev, J. Aerosol Sci. 35 (2004) 363-381.

[33] H. Kimura, I. Mann, J. Quant. Spectrosc. Radiat. Transfer 60 (1998) 425-438 